\title{
Safety Evaluation of Fog Warning Systems in a Connected Vehicle Environment Based on Sample Entropy
}

\author{
Xuewei Li, ${ }^{1}$ Yuchen Jia, ${ }^{1}$ Yufei Chen, ${ }^{2}$ Guanyang Xing, ${ }^{1}$ Xiaohua Zhao $\mathbb{D}^{1},{ }^{1}$ and Jian Rong $^{1}$ \\ ${ }^{1}$ Beijing Key Laboratory of Traffic Engineering, Beijing University of Technology, Beijing 100124, China \\ ${ }^{2}$ China Automotive Engineering Research Institute, Chongqing 401120, China \\ Correspondence should be addressed to Xiaohua Zhao; zhaoxiaohua@bjut.edu.cn
}

Received 14 July 2021; Revised 2 September 2021; Accepted 16 September 2021; Published 6 October 2021

Academic Editor: Xinqiang Chen

Copyright (c) 2021 Xuewei Li et al. This is an open access article distributed under the Creative Commons Attribution License, which permits unrestricted use, distribution, and reproduction in any medium, provided the original work is properly cited.

Changes in driving behavior caused by reduced visibility in fog can lead to crashes. To improve driving safety in fog weather, a fog warning system based on connected vehicle (CV) technology is proposed. From the perspective of human factors, this study evaluates the driving safety based on drivers' speed change under different fog levels (i.e., no fog, light fog, and heavy fog) and different technical levels (i.e., normal, with a dynamic message sign (DMS), and with a human-machine interface (HMI)). The driving behavior data were collected by a driving simulation experiment. The experimental road was divided into three zones: clear zone, transition zone, and fog zone. To quantify the change of vehicle speed comprehensively, the speed and acceleration were selected. Meanwhile, the vehicle speed safety entropy and acceleration safety entropy were proposed based on sample entropy theory. Furthermore, the changes of each index in different zones were analyzed. The results show that the use of fog warning system can improve speed stability and driving safety in fog zones and can make the driver decelerate in advance with a smaller deceleration before entering the fog zones. The higher the technical level is, the earlier the driver decelerates. Under the condition of light fog, the fog warning system with HMI has a better effect in terms of improving speed stability, while under the condition of heavy fog, there is little difference between the two technical levels. In general, this study proposed a novel safety evaluation index and a general evaluation method of the fog warning system.

\section{Introduction}

With the decrease of visibility on foggy days, the number of traffic accidents increases significantly. Statistics show that the number of crashes caused by foggy conditions in the United States is as high as $300-400$ per year [1]. In China, traffic accidents caused by foggy conditions are far more likely to cause serious consequences than other adverse weather conditions, and the number of traffic crashes caused by fog in China reached 633 in 2017 [2]. Therefore, ensuring the safety of driving on foggy days is the focus of the current research.

A large number of studies have shown that the reduced visibility in foggy weather directly led to the change of longitudinal driving behavior and affected traffic flow operations. Studies investigated the relationship between drivers' car-following behaviors and the conditions of reduced visibility and found that changes in the car-following distance and vehicle speed could reflect the driver's nervousness in foggy weather [3,4]. It was presented that fog led to a decrease in speed and acceleration, as well as an increase in distance to the lead vehicle [5]. Besides, the change in longitudinal driving behavior would increase the possibility of rear-end collisions. Muellert et al. [6] found that novice drivers had higher hazard response times, greater speed, and steering variability in fog, and they are more likely to have collisions. It can be concluded that speed adaptation and reasonable distance between adjacent cars are crucial for roadway traffic safety $[7,8]$, especially in adverse weather.

At present, the fog warning system provides a new solution to improve traffic safety in foggy weather and has received more attention in the research field. The system based on connected vehicle (CV) technology can transmit 
fog information to the driver in advance so that they can adjust their speed before entering the fog zone and keep a proper distance from the front car. Among them, vehicle-tovehicle (V2V) and vehicle-to-infrastructure (V2I) technology play a huge role in addressing traffic safety, efficiency, and green issues. The US National Highway Traffic Safety Administration [9] has estimated that up to $80 \%$ of crashes can be prevented by V2V- and V2I-related safety applications. Roadside terminals and vehicle terminals are two ways to provide driver information by using CV technology. V2I warns drivers through the dynamic message sign (DMS), and $\mathrm{V} 2 \mathrm{~V}$ warns drivers through the human-machine interface (HMI). Many researchers have tried to determine whether DMS and HMI can improve speed stability and driving safety in fog zones. For DMS, Wu et al. [10] constructed safety models with different control levels to reflect drivers' risk perception, speed adjustment, and driving safety in foggy weather under different adjustment conditions. Hassan and Abdel-Aty [11] tested drivers' compliance and drivers' satisfaction with variable speed limit (VSL) and changeable message signs (CMS) instructions in different visibility and traffic conditions and on two types of roadways. For HMI, Zhao et al. [9] analyzed the effect of HMI on the drivers' speed control under different fog concentrations based on a driving simulation, and the results showed that the driver's speed decreased when entering the fog zone. Chang et al. [10] proved that the driving safety in heavy fog conditions was significantly improved by HMI. As for the longitudinal driving behavior measurement of speed and acceleration, sample entropy is also an effective method to evaluate the complexity of the time series of speed change. The method was used to investigate the relationship between road line complexity and driver sensitivity [11], as well as judge whether the driver is fatigued [12]. As for the intelligent transportation system, $\mathrm{Ma}$ et al. [13] used sample entropy value as the clustering feature vector to evaluate the driving behavior stability. Therefore, sample entropy can be used to evaluate the driving stability and driving safety of the CV system.

In general, drivers' speed stability and driving safety in foggy weather was investigated in previous research, and the effect of different fog warning system has attracted a great deal of attention. All of these studies have shown that fog leads to changes in driving behavior and does great harm to traffic safety, while the fog warning system allows drivers to know the fog in advance and reduce driving risk. However, the difference in the effect of various fog warning systems on driving performance under different visibility conditions was not clear. Therefore, it is necessary to describe the changes in drivers' longitudinal behavior under different technical levels in detail to further evaluate the effectiveness and applicability of different systems.

To address the above question, this paper uses the CV test platform to evaluate the drivers' speed stability and driving safety at different technical levels under different fog levels. The driving simulator was widely used to collect the driving data and evaluate CV warning systems [10, 14, 15]; it is an ideal solution to handle the uncontrollable factors in foggy weather and evaluate the systems without the risk of crashes. To comprehensively quantify the change of vehicle operation, the vehicle speed and acceleration were selected, and the indexes of vehicle speed safety entropy and acceleration safety entropy are proposed based on sample entropy. Moreover, this paper applies the concept of grading assessment to analyze the indexes in driving safety and speed stability in different zones (i.e., whole zone, clear zone, transition zone, and fog zone). The method proposed in the paper can provide a reference for the optimization design of fog warning systems, evaluation indicators, and general evaluation methods for the safety assessment of a fog warning system.

\section{Methods}

2.1. Connected Vehicle Testing Platform. The CV test platform was constructed based on driving simulation technology (Figure 1). The test platform consists of a driving simulator, a data management center, a V2V terminal, and a V2I terminal [14]. The driving simulator system consists of a real car, computers, videos, and audio equipment. The scenario is projected onto three big screens, which provides a $130^{\circ}$ field of view; the screen resolution of the driving simulator is $1920 \times 1080$; and the simulator records the operating data (e.g., braking force, acceleration, speed, lateral placement, number of the lanes, and turning angle of the steering wheel) in $30 \mathrm{~Hz}$.

The study used the driving simulator to develop a 3D scene to simulate the fog environment and a fog warning system, as shown in Figure 1(a). Part I is the driving simulator system where the Application Programming Interface (API) was used to collect weather and distance data. Interconnections were structured between the driving simulator and driving simulator system through the interface for data collection. The virtual visibility sensor and distance sensor collected data as well. Part II shows how the distance and visibility information is corresponded and transmitted synchronously through the User Datagram Protocol (UDP) between the management center, driving simulator system, and management center. Part III is the display terminal, including a V2I terminal (DMS) and a V2V terminal (HMI). The management center sends the final display information to DMS and HMI by comparing it with the threshold value. If the sensor detects a change in data, the process would be roll-back.

2.1.1. V2I Terminal: DMS Design. The DMS design of the V2I terminal is shown in Figure 1(b). In this paper, four DMSs are set on the gantry before the fog zone, and the set interval of the DMSs is $500 \mathrm{~m}$ with reference to the rules of the navigation. In the warning state, the DMS selects the yellow font to display the level of the fog and the distance from the fog zone. In the normal state, that is, no fog state, the DMS uses green font and displays "Welcome to the highway" [15].

2.1.2. V2V Terminal: HMI Design. According to the existing onboard interface layout [16], the HMI of the warning system includes four parts, as shown in Figure 1(c). 


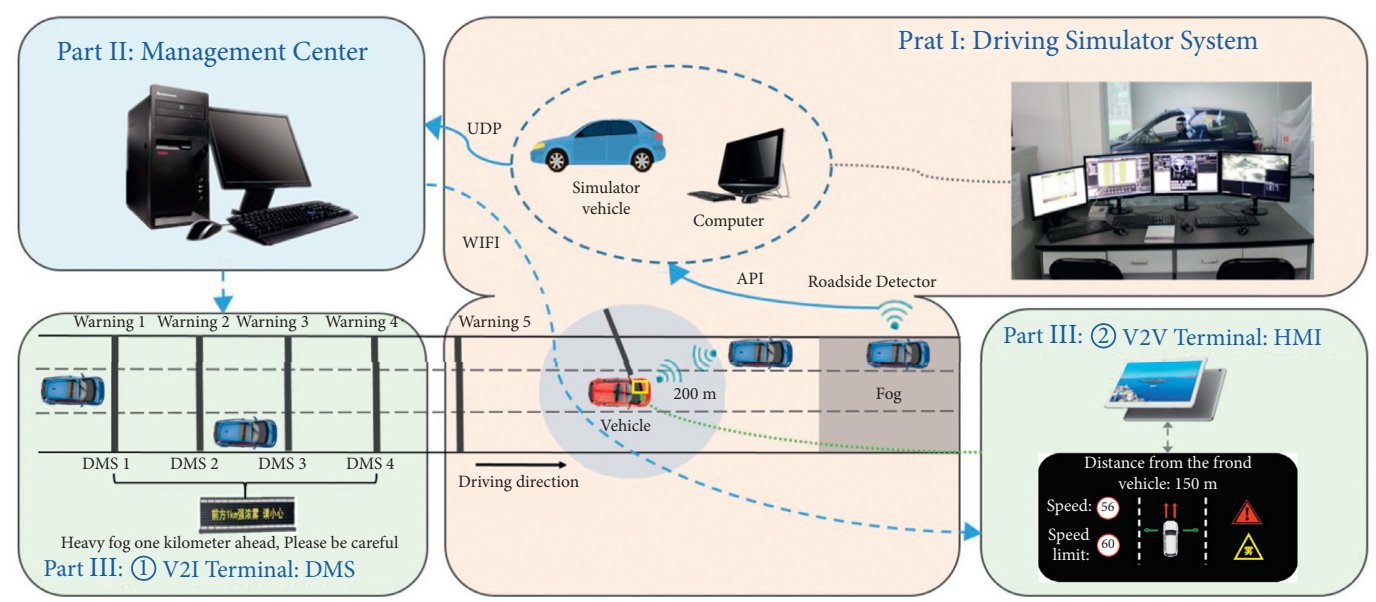

(a)

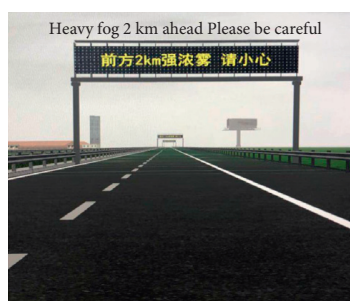

DMS 1

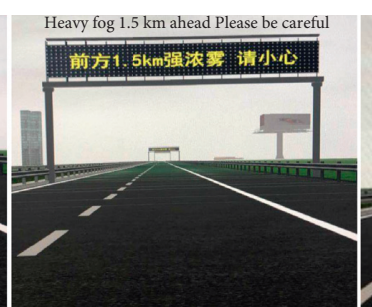

DMS 2

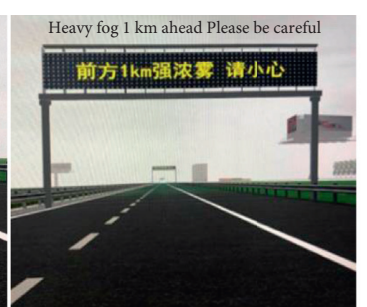

DMS 3

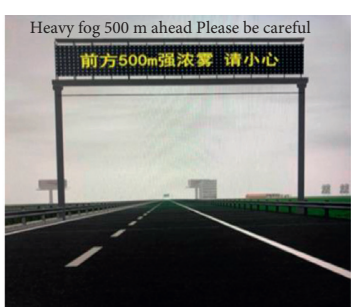

DMS 4

(b)

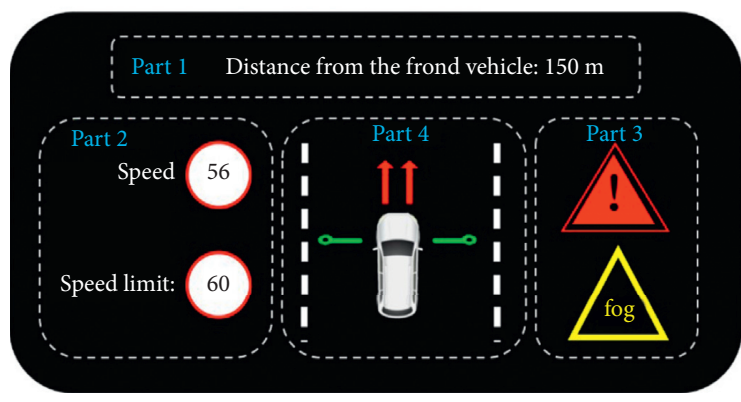

The layout of the HMI

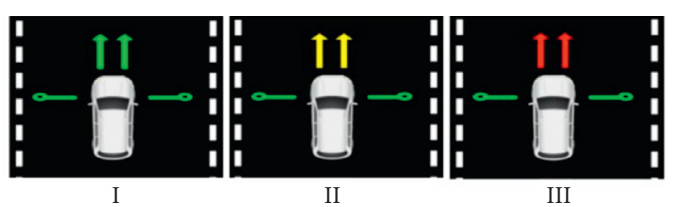

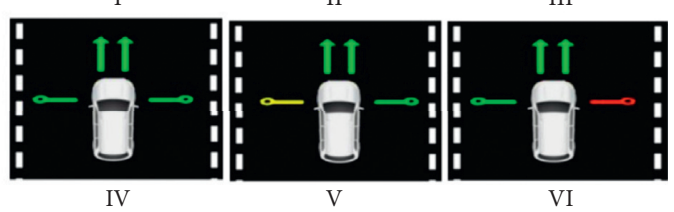

Different situations

(c)

FIGURE 1: Driving simulation platform and terminal design. (a) Driving simulation platform, (b) vehicle-to-infrastructure (V2I) terminal, dynamic message sign (DMS), and (c) vehicle-to-vehicle (V2V) terminal, and human-machine interface (HMI).

Part 1 displays the distance from the preceding vehicle in the normal state. In the warning state, the HMI includes five warning points with voice prompts in total. The first four warning points are consistent with the information displayed by the DMS, and their actual location is within the first $50 \mathrm{~m}$ of each DMS, while the fifth warning point is set at the beginning of the fog zone to remind drivers that they have entered the fog zone.

Part 2 is the speed prompt zone, which includes the driving speed of the vehicle and the speed limit. The speed limit is $120 \mathrm{~km} / \mathrm{h}$ under no fog or light fog conditions and is $60 \mathrm{~km} / \mathrm{h}$ under heavy fog conditions [17]. If the drivers' speed exceeds the speed limit, the voice prompt will remind the driver: "you are speeding, please slow down."
Part 3 is the graphical prompt zone. As an auxiliary prompt, the red exclamation mark indicates that the lead vehicle is below the $2 \mathrm{~s}$ threshold [18].

Part 4 is the reminder of the surrounding vehicles. The green arrow indicates that the distance from the surrounding vehicles is greater than $200 \mathrm{~m}$. The yellow flashing arrow indicates that the distance from the surrounding vehicles is less than $200 \mathrm{~m}$. The red flashing arrow indicates that there will be a collision with the vehicle ahead of less than $2 \mathrm{~s}$ at the current speed.

This CV platform realizes the human-computer interaction of the fog warning system and evaluates the adaptability and driving safety indexes. The platform lays the foundation for the specific practical application of an early warning system. 
2.2. Participants. The experiment invited 43 participants, including 28 males (age: $37.5 \pm 13.1$ years, driving years: $16 \pm 10.2$ years, and mean annual driving mileage: $18,524 \pm 3548.22 \mathrm{~km}$ ) and 15 females (age: $25 \pm 12.97$ years, driving years: $13 \pm 9.3$ years, and mean annual driving mileage: $9,584 \pm 5514.21 \mathrm{~km}$ ). The participants were recruited from college and taxi companies, and all of them have more than 3 years of driving experience. To statistically examine whether the sample was sufficient for this study, a power analysis was conducted using equation (1). The method is shown in the following equation:

$$
n=\frac{\left(z_{\alpha / 2}+z_{\beta}\right)^{2} \sigma^{2}}{\varepsilon^{2}}
$$

where $z_{\alpha / 2}$ is the upper $(\alpha / 2)$ th quantile of the standard normal distribution; $z_{\beta}$ is the upper $(\beta)$ th quantile of the standard normal distribution; $\sigma$ is the standard deviation of the normal distribution population; $\varepsilon$ is the difference between the true mean response of a test factor and a reference value, which can be given by $\varepsilon= \pm \delta \sigma$ [19]; $\delta$ is the meaningful difference. In practice, a value between 0.25 and 0.5 is usually chosen as $\delta$ if there is no prior knowledge [20]. Typically, a $10 \%$ level of significance is chosen to reflect a $90 \%$ confidence regarding the unknown parameter. A power of $80 \%$ and a meaningful difference of 0.5 were used to balance the power and cost-effectiveness. The results showed that the required sample size in this research was 25 . This implied that the experiment design could provide reliable answers to the questions to be investigated.

2.3. Scenario Design. In this study, nine scenarios $(3 \times 3)$ are designed based on two variables (visibility levels and technical levels). The three levels of visibility are no fog, light fog, and heavy fog, and the three technical levels are normal (named group (1)), with DMS (named group (2)), and with HMI (named group (3)). The detailed information of each group in the experimental scene design is shown in Table 1.

The test road is designed according to the main corridor of the 2022 Winter Olympic Games. The cross section of the road was $26 \mathrm{~m}$ (four lanes with lane width $=3.75 \mathrm{~m}$, median width $=2 \mathrm{~m}$, and shoulder-width $=4.5 \mathrm{~m}$ ) and a speed limit of $60-120 \mathrm{~km} / \mathrm{h}$ on a normal road [17] (Figure 2).

In the experimental design, this study divided the road into four zones (Figure 2(a)): (1) speed up zone $(1.5 \mathrm{~km})$, the function of which is to stabilize the driver's speed; (2) clear zone $(1.5 \mathrm{~km})$, which is used to give drivers the information about fog; (3) transition zone $(0.5 \mathrm{~km})$, the role of which is to make the drivers adapt to the change of visibility; and (4) fog zone $(2 \mathrm{~km})$, which was employed to compare driving behavior data in different visibility conditions.

For traffic flow, since fog usually appears in the morning and the traffic volume is small in the morning, this study selects free-flow traffic with an average headway of $36 \mathrm{~s}$, and the average speed of other vehicles is $100 \mathrm{~km} / \mathrm{h}$ under no fog and light fog conditions and $55 \mathrm{~km} / \mathrm{h}$ under foggy condition.

The fog level classification design in the experiment was based on China's Fog Classification (GBT 27964-2011)"
TABLE 1: Detailed information of each group in experimental scene design.

\begin{tabular}{lccc}
\hline Group & Traffic flow & Technical level & Fog level \\
\hline Group (1) & Free-flow & Normal & $\begin{array}{c}\text { No fog } \\
\text { Light fog } \\
\text { Heavy fog }\end{array}$ \\
\hline Group (2) & Free-flow & DMS & $\begin{array}{c}\text { No fog } \\
\text { Light fog } \\
\text { Heavy fog }\end{array}$ \\
\hline Group (3) & Free-flow & HMI & $\begin{array}{c}\text { No fog } \\
\text { Light fog } \\
\text { Heavy fog }\end{array}$ \\
\hline
\end{tabular}

[21], and the rendering characteristics of the simulator were also taken into account to create significant visual differences among different fog visibility levels. In this paper, three visibility levels were defined as no fog, light fog, and heavy fog, and the visibility is $10000 \mathrm{~m}, 750 \mathrm{~m}$, and $125 \mathrm{~m}$, respectively (Figure 2(b)).

2.4. Experimental Procedure. The driving simulation experiment steps employed in this study are as follows:

(1) Filling in the questionnaire before driving. The demographic information and fatigue degree of the participants were recorded before driving.

(2) Predriving training. Through predriving training, participants were able to learn to correctly use and understand the HMI and DMS.

(3) Driving practice. The purpose of the driving practice was to familiarize the drivers with the driving simulator.

(4) Formal experiment. The participants completed the experimental scenes in random order. To avoid fatigue, participants took at least five minutes off between each experiment.

(5) Filling in the questionnaire after driving. The purpose of filling in the questionnaire after driving was to get the driver's after-driving fatigue degree and subjective feelings for the experiment.

2.5. Data Processing and Indicator Selection. In the experiment, the data collection range in the whole zone was from the DMS1 first $200 \mathrm{~m}$ to the end of the fog zone (point A to point D in Figure 2(a)). The data collection zones were the clear zone (A to $B$ ), the transition zone (B to $C$ ), and the fog zone (C to D). MATLAB was used to intercept data every $20 \mathrm{~m}$. This study collected the effective data of 43 drivers in nine scenes, and a total of 387 sets of data were used to analyze the effectiveness of fog warning systems. The speed stability of the driver in the fog area is important for the evaluation of the effect of a fog warning system, and speed stability is an alternative indicator of safety. In this study, two indicators were selected to represent driving stability and safety: 


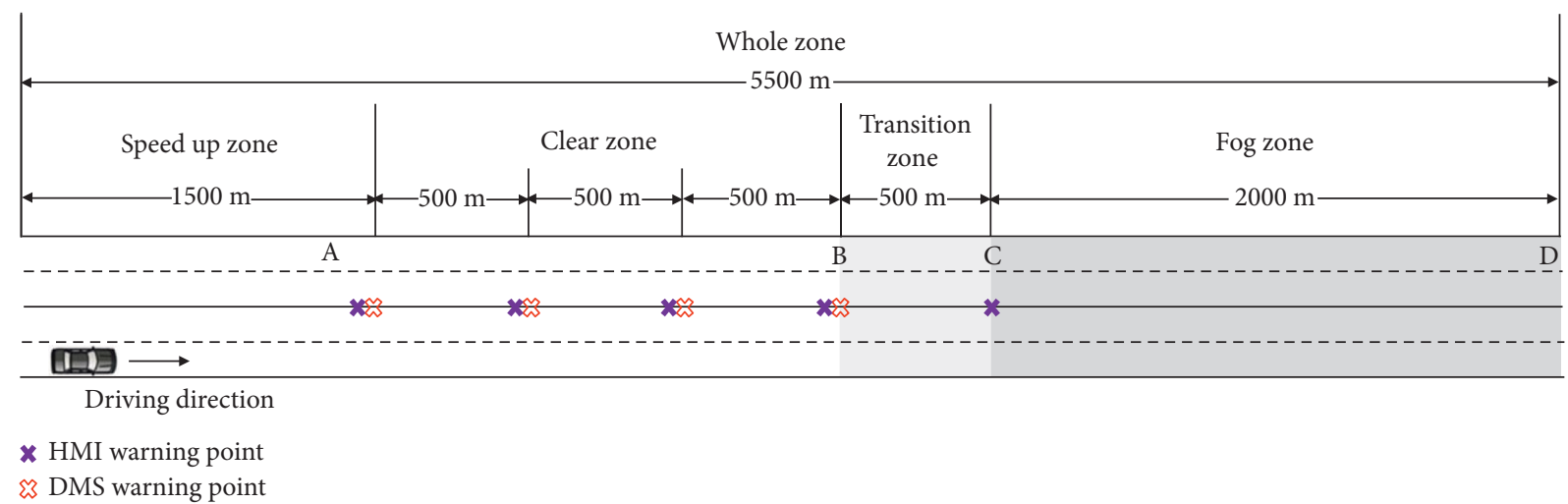

(a)

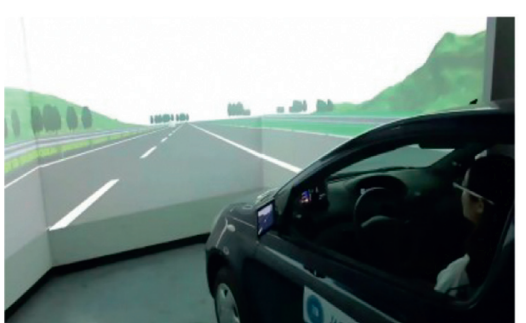

No fog

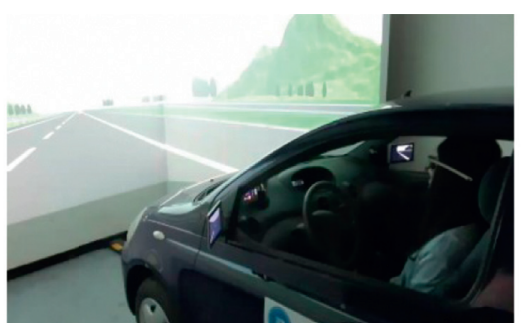

Light fog

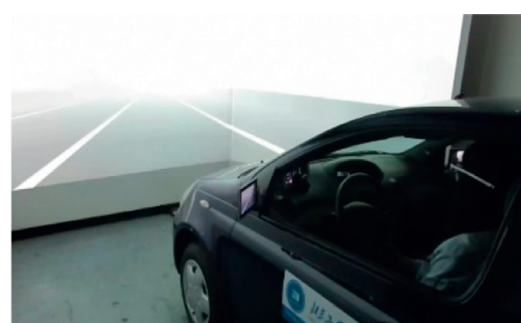

Heavy fog

(b)

Figure 2: Scenario and fog level design. (a) Scenario design. (b) Different visibilities in the fog zone.

(1) Speed is the most intuitive indicator to reflect the driving state on the road. The smaller the speed, the higher the safety of the driver.

(2) Acceleration was used to represent how fast the driver's speed changed. It is the ratio of the amount of speed change to the time it takes to reflect on the change in speed. It is one of the most important indicators of driving safety.

These two indicators can represent the speed stability and driving safety of the driver under conditions of different technical levels and fog levels.

\section{Results}

Firstly, the basic trend changes of the speed and acceleration were obtained through a pairwise comparison analysis of different factors and levels. Since fog level is a categorical variable and there is no correlation between the three fog levels, therefore, one-way analysis of variance (ANOVA) method was applied to analyze the significance of the index difference in different zones. Secondly, to further understand the differences of various indicators under different technical levels, the least-significant difference (LSD) was used to test the intergroup significance difference. Finally, to comprehensively quantify speed fluctuations, this study proposes the speed safety entropy and acceleration safety entropy based on the sample entropy method. The chaotic degree of speed change of the driver after entering the fog area (clear zone) and the visibility change (transition zone and fog zone) and finally the significance of the index difference between different zones were analyzed by one-way ANOVA.

\subsection{Statistical Analysis}

3.1.1. No Fog. Speed and acceleration were used to analyze the trend of a driver's driving behavior change in the clear zone, the transition zone, and the fog zone. The results of the significance of the index difference and the mean values and standard deviation values of the indexes in no fog condition in each zone are shown in Table 2. The speed and acceleration variation trends are shown in Figure 3.

In the whole zone, the LSD test results of speed show that there is a significant difference $(P=0.025)$ between group (1) and the other groups in the whole zone. Specifically, group (1) has the largest acceleration and the lowest average speed, and group (3) has the smallest overall acceleration value. As shown in Figure 3, the drivers' speed change trend in group (3) and group (2) is almost simultaneous, but the acceleration of group (3) was significantly lower in the transition zone.

The above results show that, in the state of no fog, the driver's speed control ability can be improved with the increase of the technical level, and the driving process can be smoother.

3.1.2. Light Fog. In the case of light fog, the mean speed of group (2) and group (3) in the whole zone is lower than that of group (1) and also has higher acceleration. Significant differences among the three groups' speed in clear zone, transition 
TABLE 2: Statistical analysis of index under no fog condition.

\begin{tabular}{|c|c|c|c|c|c|c|c|}
\hline \multirow{2}{*}{ Zone } & \multirow{2}{*}{ Group } & \multicolumn{3}{|c|}{ Speed $(\mathrm{km} / \mathrm{h})$} & \multicolumn{3}{|c|}{ Acceleration $\left(\mathrm{m} / \mathrm{s}^{2}\right)$} \\
\hline & & Mean (SD) & $P$ & Difference between groups & Mean (SD) & $P$ & Difference between groups \\
\hline \multirow{3}{*}{ Whole zone } & Group (1) & $105.672(4.690)$ & \multirow{3}{*}{$0.011^{*}$} & \multirow{3}{*}{ (1)-(2) } & $0.195(0.097)$ & & \multirow{3}{*}{ (1)-(3) } \\
\hline & Group (2) & $107.265(5.757)$ & & & $0.183(0.105)$ & $0.025^{*}$ & \\
\hline & Group (3) & $106.443(5.851)$ & & & $0.139(0.104)$ & & \\
\hline \multirow{3}{*}{ Clear zone } & Group (1) & $100.452(2.943)$ & \multirow{3}{*}{$0.017^{*}$} & \multirow{3}{*}{ (1)-(2) } & $0.202(0.115)$ & & \multirow{3}{*}{ (1)-(3) } \\
\hline & Group (2) & $102.869(6.577)$ & & & $0.249(0.131)$ & $0.011^{*}$ & \\
\hline & Group (3) & $101.253(6.355)$ & & & $0.187(0.108)$ & & \\
\hline \multirow{3}{*}{ Transition zone } & Group (1) & $108.192(4.645)$ & \multirow{3}{*}{$\leq 0.001^{*}$} & (1)-(2) & $0.120(0.100)$ & \multirow{3}{*}{$0.001^{*}$} & \multirow{3}{*}{$\begin{array}{l}\text { (1)-(3) } \\
\text { (2)-(3) }\end{array}$} \\
\hline & Group (2) & $111.727(6.581)$ & & (1)-(3) & $0.164(0.139)$ & & \\
\hline & Group (3) & $109.499(3.417)$ & & (2) - (3) & $0.103(0.009)$ & & \\
\hline \multirow{3}{*}{ Fog zone } & Group (1) & $109.322(4.937)$ & \multirow{3}{*}{$0.013^{*}$} & (1)-(2) & $0.209(0.139)$ & \multirow{3}{*}{0.612} & \multirow{3}{*}{-} \\
\hline & Group (2) & $109.804(5.410)$ & & (1)-(3) & $0.137(0.113)$ & & \\
\hline & Group (3) & $109.946(3.913)$ & & & $0.126(0.095)$ & & \\
\hline
\end{tabular}

*Significant at the $95 \%$ confidence level.

zone, and fog zone were observed, as shown in Table 3 and Figure 4. Specifically, in the clear zone, the speed of group (1) increases slowly and continuously, group (2) and group (3) start to accelerate after passing the first warning point. In the transition zone, due to the insignificant change in visibility, the speed of group (1) is still increasing, but group (2) and group (3) start to slow down until the speed in the fog zone stabilizes. After entering the fog zone, due to changes in visibility, group (1) began to slow down. In the latter part of the fog zone, after the three groups of speeds stabilized, the speed difference is not large. It can be seen that drivers in group (2) kept a lower average speed in the transition zone. However, drivers in group (3) were the first group to slow down in the transition zone and then kept a lower average speed in the fog zone (Figure 4).

The above results show that, in light fog, the groups with a warning system slow down in advance, and the higher the technical level is, the earlier the driver slows down. Drivers can better adjust their speeds and can be more adaptable to the visibility change. Besides, the HMI helps drivers to keep lower average speed in light fog zone.

3.1.3. Heavy Fog. In the heavy fog condition, the mean speed of group (1) in the whole zone is greater than that of group (2) and group (3), and the average speed in subzones was lower than that in light fog, as shown in Table 4 and Figure 5. In the clear zone, group (2) and group (3) were in a deceleration state when the warning information of the first and the fourth warnings was received. The probable reason for this deceleration may be that the warning information of heavy fog has an impact on the driver at the first warning point and thus produces a deceleration state. However, due to the great distance from the fog zone, group (2) and group (3) gradually tended to display a uniform speed and accelerated after a period of deceleration, until the drivers received warning information that they were $500 \mathrm{~m}$ from the heavy fog zone, at which point they started to slow down continuously. In the transition zone, group (3) was the first to slow down, followed by group (2) with reduced visibility. On the verge of reaching the fog zone, the deceleration in group (1) began to increase. At the beginning of the fog zone, with a significant reduction in visibility, the deceleration in group (1) was significantly increased, and after the continuous slowing down, their deceleration began to gradually decrease. In the second half of the fog zone, after the driver's speed reached a stable value, there was no obvious difference in the acceleration change. In the changing process, a significant difference of acceleration was found between group (2) and group (3) in the transition zone. Drivers in group (3) decreased earlier and greater. In addition, drivers in group (2) kept a lower average speed than that of group (3) in the heavy fog zone.

The above results show that, in heavy fog, the fog warning system (i.e., HMI and DMS) can make the driver deceleration smoother and allow the driver to decelerate ahead of time before the visibility changed. Driver decelerates more early in heavy fog condition than light fog condition. Drivers using HMI decreased earlier and greater in the transition zone, and divers kept lower average speed in the heavy fog zone than that using DMS.

\subsection{Safety Analysis of a Fog Warning System Based on Sample Entropy}

3.2.1. Sample Entropy. To comprehensively quantify speed stability and driving safety, this paper introduces the method of sample entropy to quantify the impact of a fog warning system on foggy driving safety.

Sample entropy is a method to detect the complexity of time series by quantifying the degree of uncertainty, the time series complexity is obtained by measuring the probability of generating a new pattern in the signal, and the greater the probability that a new pattern is generated, the greater the complexity of the sequence is [17]. Sample entropy represents the entropy rule under time series changes. Because sample entropy can simplify the quantification of complex signals, it is an effective tool for detecting changes in driving behavior rules in a short time [22-24]. Sample entropy has unique advantages in evaluating driving behavior data. On the one hand, sample entropy can be used to reflect the uncertainty of the driver's time series behavior. On the other hand, when there are multiple peaks in the data, the mean 


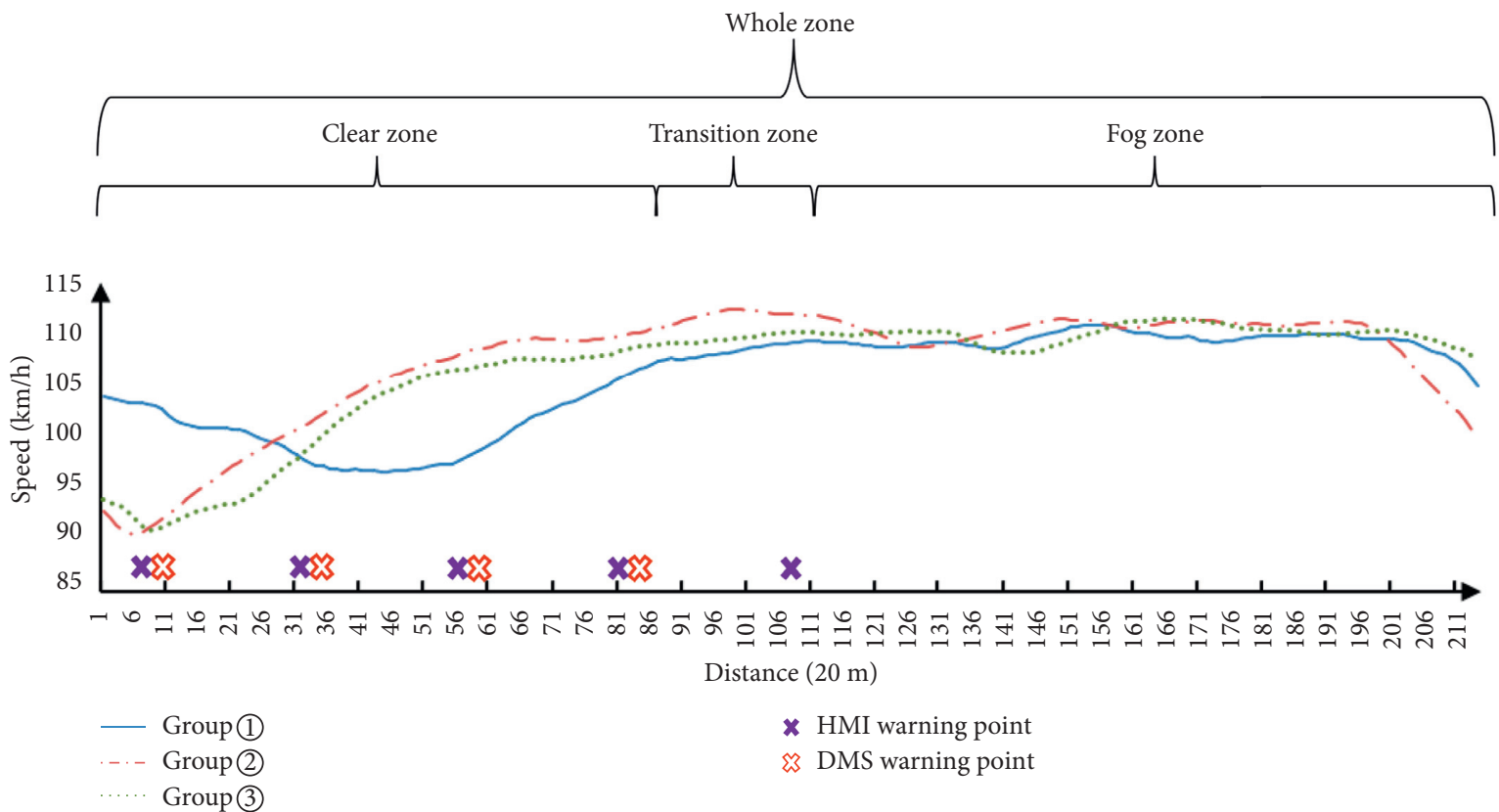

(a)

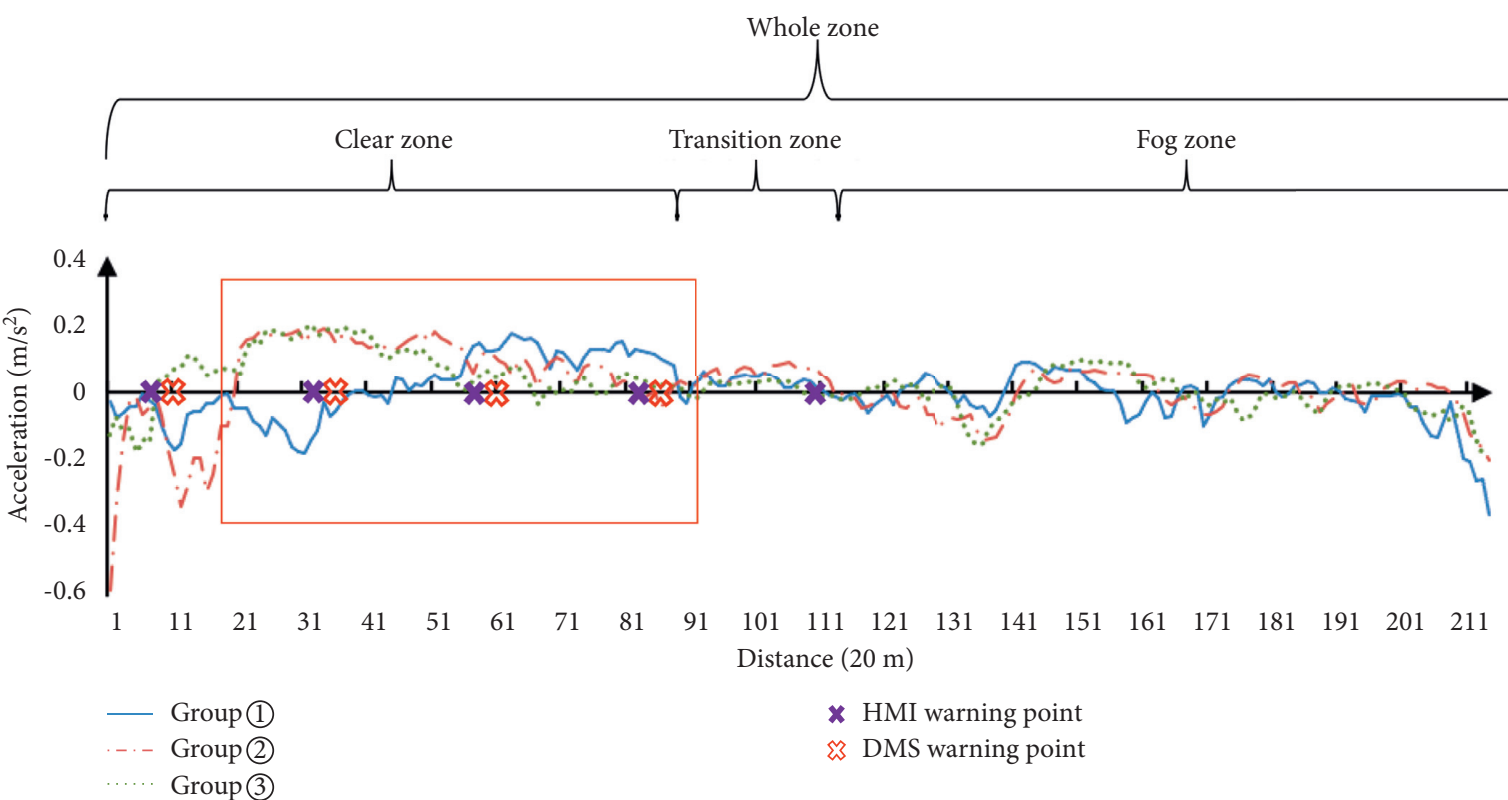

(b)

Figure 3: Speed and acceleration in no fog. (a) Speed and (b) Acceleration.

and variance of the indicators tend to ignore the variability of the data, and entropy can more fully describe the changes in the indicators. Therefore, this study chooses the method of sample entropy to characterize the fluctuation of safety indicators, that is, to quantify the impact of technical level on driving safety and speed stability and uniformly name the sample entropy that characterizes the safety index as safety entropy. The entropy value is small when the data are concentrated, while the value is high when the data are scattered. The calculation process of sample entropy realized by MATLAB programming is as follows:
(1) Let the original data be $x$ (1) and $x$ (2), for a total of $N$ numbers.

(2) Make up the $m$ vector, and subtract every two vectors (equation (2)):

$$
\begin{aligned}
X(i) & =[x(i), x(i+1), \ldots, x(i+m-1)] ; \\
i & =j=1,2, \ldots, N-m+1, \ldots,
\end{aligned}
$$

where $m$ is the embedded dimension and $N$ represents the data length. Normally, $m$ is taken as 1 or 2 . When $m>2$, the sample demand increases greatly. In this study, $m$ was chosen to be 2 , and the value of $\mathrm{N}$ 
Table 3: Statistical analysis of index under light fog condition.

\begin{tabular}{|c|c|c|c|c|c|c|c|}
\hline \multirow{2}{*}{ Zone } & \multirow{2}{*}{ Group } & \multicolumn{3}{|c|}{ Speed $(\mathrm{km} / \mathrm{h})$} & \multicolumn{3}{|c|}{ Acceleration $\left(\mathrm{m} / \mathrm{s}^{2}\right)$} \\
\hline & & Mean (SD) & $P$ & Difference between groups & Mean (SD) & $P$ & Difference between groups \\
\hline \multirow{3}{*}{ Whole zone } & Group (1) & $106.032(7.958)$ & & (1)-(2) & $0.207(0.185)$ & & (1)-(2) \\
\hline & Group (2) & $92.625(6.375)$ & $\leq 0.001^{*}$ & (1)-(3) & $0.218(0.124)$ & $\leq 0.001^{*}$ & (1)-(3) \\
\hline & Group (3) & $93.585(6.718)$ & & & $0.238(0.224)$ & & \\
\hline \multirow{3}{*}{ Clear zone } & Group (1) & $111.363(2.823)$ & & (1)-(2) & $0.130(0.090)$ & & (1)-(2) \\
\hline & Group (2) & $87.692(6.696)$ & $\leq 0.001^{*}$ & (1)-(3) & $0.309(0.121)$ & $0.011^{*}$ & (2)-(3) \\
\hline & Group (3) & $91.063(8.424)$ & & (2)-(3) & $0.290(0.101)$ & & \\
\hline \multirow{3}{*}{ Transition zone } & Group (1) & $115.647(6.542)$ & & (1)-(2) & $0.289(0.170)$ & & - \\
\hline & Group (2) & $98.225(7.229)$ & $\leq 0.001^{*}$ & (1)-(3) & $0.201(0.179)$ & 0.063 & \\
\hline & Group (3) & $102.302(5.646)$ & & (2)-(3) & $0.220(0.159)$ & & \\
\hline \multirow{3}{*}{ Fog zone } & Group (1) & $99.520(6.001)$ & & (1)-(2) & $0.201(0.141)$ & & (1)-(2) \\
\hline & Group (2) & $95.339(3.459)$ & $\leq 0.001^{*}$ & (1)-(3) & $0.143(0.106)$ & $\leq 0.001^{*}$ & (1)-(3) \\
\hline & Group (3) & $93.634(3.454)$ & & (2)-(3) & $0.205(0.139)$ & & (2)-(3) \\
\hline
\end{tabular}

*Significant at the $95 \%$ confidence level.

was selected to be within the range of $100-5000$, as 1000.

(3) Define the distance $d_{x(i), x(j)}$ between the vectors $x(i)$ and $x(j)$ (equation $(3)$ ):

$$
\begin{aligned}
d_{x(i), x(j)} & =\max |x(i+k)-x(j+k)|, \\
k & =0,1,2, \ldots, m+1,
\end{aligned}
$$

where $d_{x(i), x(j)}$ is defined as the maximum distance parameters of the difference between elements $x(i)$ and $x(j)$.

(4) The parameter $m$ can be obtained by equations (4) and (5):

$$
\begin{aligned}
B_{i m}(r) & =\frac{\operatorname{Num}\left[d_{x(i), x(j)}<r\right]}{N-m+1}, \\
B_{m}(r) & =\frac{1}{N-m+1} \sum_{i=1}^{N-m+1} B_{i m}(r),
\end{aligned}
$$

where $B_{i m} I$ is the ratio of the number of times that $d_{x(i), x(j)}$ is less than $r$ and the total number of distances $N-m+1, r$ is the tolerance error of similarity, and the value of $r$ is generally as expressed in the following equation:

$$
r=0.1 \sim 0.25 E
$$

where $E$ is the variance of the original data, $0.1 e$ or $0.25 e$. In this study, $r=0.25 e$ was selected.

(5) Add 1 to the parameter dimension and repeat the above steps to get $B_{m+1}(r)$, when $n<\infty$ (equation (7)):

$$
S(m, r, N)=-\ln \left[\frac{\left(B_{m+1}(r)\right)}{B_{m}(r)}\right],
$$

where $S(m, r, N)$ express sample entropy.

In this study, the two indexes of speed and acceleration were selected to calculate sample entropy, named speed safety entropy and acceleration safety entropy. To analyze the speed stability and driving safety of the driver on a foggy day with more details by safety entropy, the safety entropy was divided into two types: before entering the fog zone (clear zone) and after visibility changes (transition zone and fog zone). Because the focus of the study is to study the effect of technical level on driving behavior in different foggy days, a one-way ANOVA method was selected for sample entropy for comparison within the group.

3.2.2. No Fog. The speed safety entropy and acceleration safety entropy were analyzed to evaluate the speed change of drivers under different technical and fog levels, and the results are shown in Figure 6.

In terms of the speed safety entropy under the condition of no fog (Figure 6(a)), due to no warning information, there is no significant difference in the speed safety entropy among the three groups. The value range and average value of group (3) are the smallest. As for the acceleration (Figure 6(b)), there is little difference in the mean value of acceleration safety entropy among the three groups, and the overall safety entropy range is slightly lower in group (3). The results show that, in the case of no danger (no fog), the road information and surrounding vehicle information provided by HMI help drivers to drive in a more stable state.

3.2.3. Light Fog Condition. In terms of the speed safety entropy under the condition of light fog (Figure 7(a)), in the clear zone, there are significant differences between group (1) and group (2), while the difference between group (2) and group (3) is relatively small. In the transition zone and fog zone, the range of the average speed safety entropy of the group (2) was larger than the other two groups, which means the speed change of drivers in group (2) is more complex and unordered, and therefore, the speed stability would be affected. Therefore, drivers using DMS performed more stable speed in clear zone, while drivers using HMI performed better speed stability after the visibility changes.

In terms of the acceleration safety entropy (Figure 7(b)), in the clear zone, due to the influence of warning information, there are significant differences between group (1) and group (2) and group (2) and group (3). The safety entropy range of 


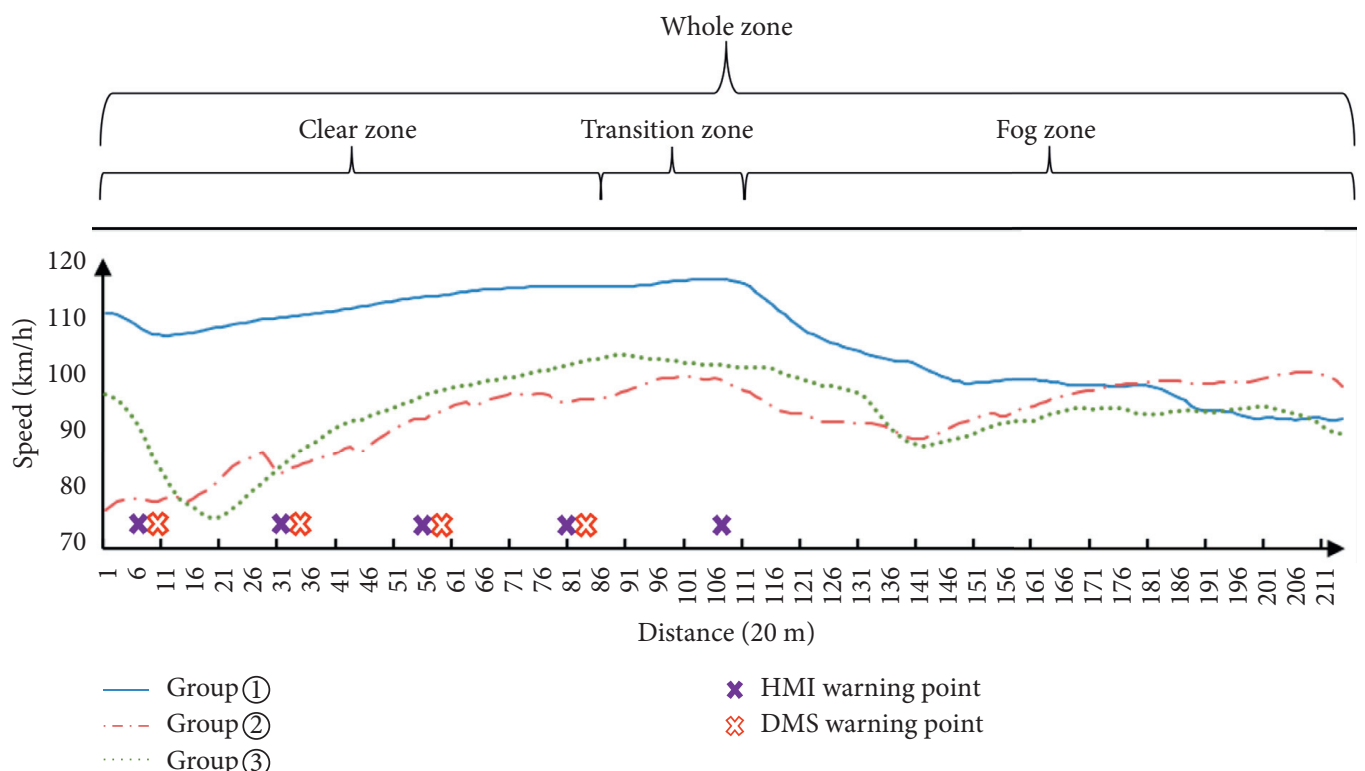

(a)

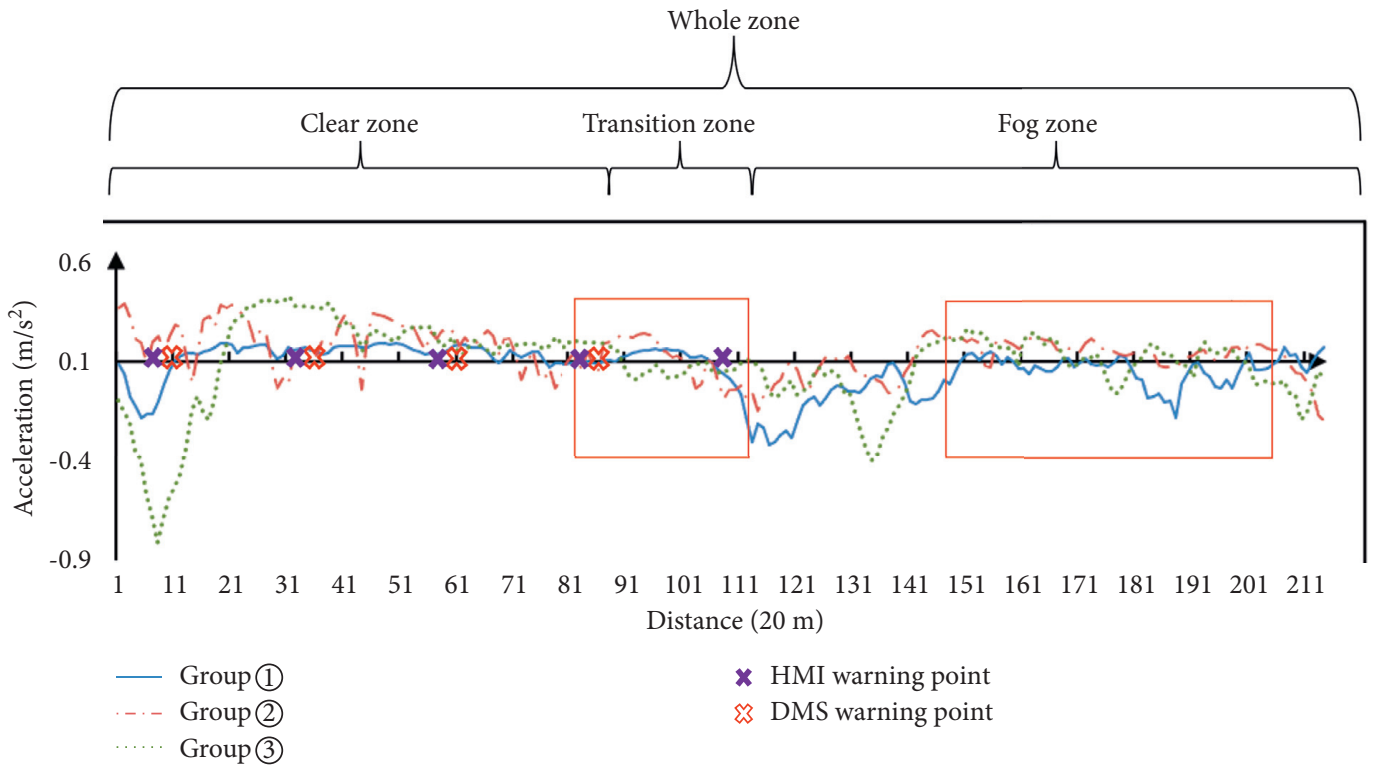

(b)

Figure 4: Light fog. (a) Speed and (b) acceleration.

group (2) and group (3) is larger than that of group (1), which indicated that the drivers responded to the warning information and were more active to adjust the speed constantly. However, in the transition zone and the fog zone, there is little difference in the safety entropy of group (2) and group (3). The safety entropy of most drivers in group (2) and group (3) is lower than in group (1), but for a small number of drivers in group (2) and group (3), the entropy of safety is significantly too high. This indicates that the fog warning system has positive effects on the improvement of driving safety of most drivers, but a small number of drivers may not correctly understand or adapt to the fog warning system, causing large fluctuations in speed.
3.2.4. Heavy Fog. As for the speed safety entropy (Figure 8(a)), the driver has a greater speed safety entropy than that in light fog, which indicates that the time series change of vehicle speed is more complex in heavy fog. In the clear zone, according to the results of the one-way ANOVA, there are significant differences between group (1) and group (2), the difference between group (1) and group (3) is small, and the mean speed safety entropy of group (2) and group (3) is less than group (1). In the transition zone and fog zone, one-way ANOVA showed that there were no significant differences between the three groups, the difference in speed safety entropy values between group (1) and group (2) is small, and the speed safety entropy of group (3) is smaller than other groups. The complexity of driver's speed 
TABLE 4: Statistical analysis of index under heavy fog condition.

\begin{tabular}{|c|c|c|c|c|c|c|c|}
\hline \multirow{2}{*}{ Zone } & \multirow{2}{*}{ Group } & \multicolumn{3}{|c|}{ Speed $(\mathrm{km} / \mathrm{h})$} & \multicolumn{3}{|c|}{ Acceleration $\left(\mathrm{m} / \mathrm{s}^{2}\right)$} \\
\hline & & Mean (SD) & $P$ & Difference between groups & Mean (SD) & $P$ & Difference between groups \\
\hline \multirow{3}{*}{ Whole zone } & Group (1) & $88.854(14.358)$ & & (1)-(2) & $0.274(0.134)$ & & \\
\hline & Group (2) & $82.068(10.248)$ & $\leq 0.001^{*}$ & (1)-(3) & $0.255(0.124)$ & 0.469 & - \\
\hline & Group (3) & $82.393(13.452)$ & & & $0.353(0.144)$ & & \\
\hline \multirow{3}{*}{ Clear zone } & Group (1) & $103.267(6.143)$ & & (1)-(2) & $0.245(0.092)$ & & (1)-(2) \\
\hline & Group (2) & $99.737(10.432)$ & $\leq 0.001^{*}$ & (1)-(3) & $0.163(0.112)$ & $\leq 0.001^{*}$ & (1)-(3) \\
\hline & Group (3) & $98.481(10.895)$ & & (2)-(3) & $0.186(0.119)$ & & \\
\hline \multirow{3}{*}{ Transition zone } & Group (1) & $107.230(8.223)$ & & (1)-(2) & $0.235(0.191)$ & & (1)-(3) \\
\hline & Group (2) & $92.157(10.358)$ & $\leq 0.001^{*}$ & (1)-(3) & $0.318(0.254)$ & $\leq 0.001^{*}$ & (2)-(3) \\
\hline & Group (3) & $94.319(13.514)$ & & & $0.657(0.364)$ & & \\
\hline \multirow{3}{*}{ Fog zone } & Group (1) & $70.433(10.342)$ & & (1)-(2) & $0.341(0.134)$ & & (1)-(2) \\
\hline & Group (2) & $64.767(13.254)$ & $\leq 0.001^{*}$ & (1)-(3) & $0.284(0.173)$ & $0.001^{*}$ & (1)-(3) \\
\hline & Group (3) & $66.643(9.845)$ & & (2)-(3) & $0.271(0.157)$ & & \\
\hline
\end{tabular}

* Significant at the $95 \%$ confidence level.

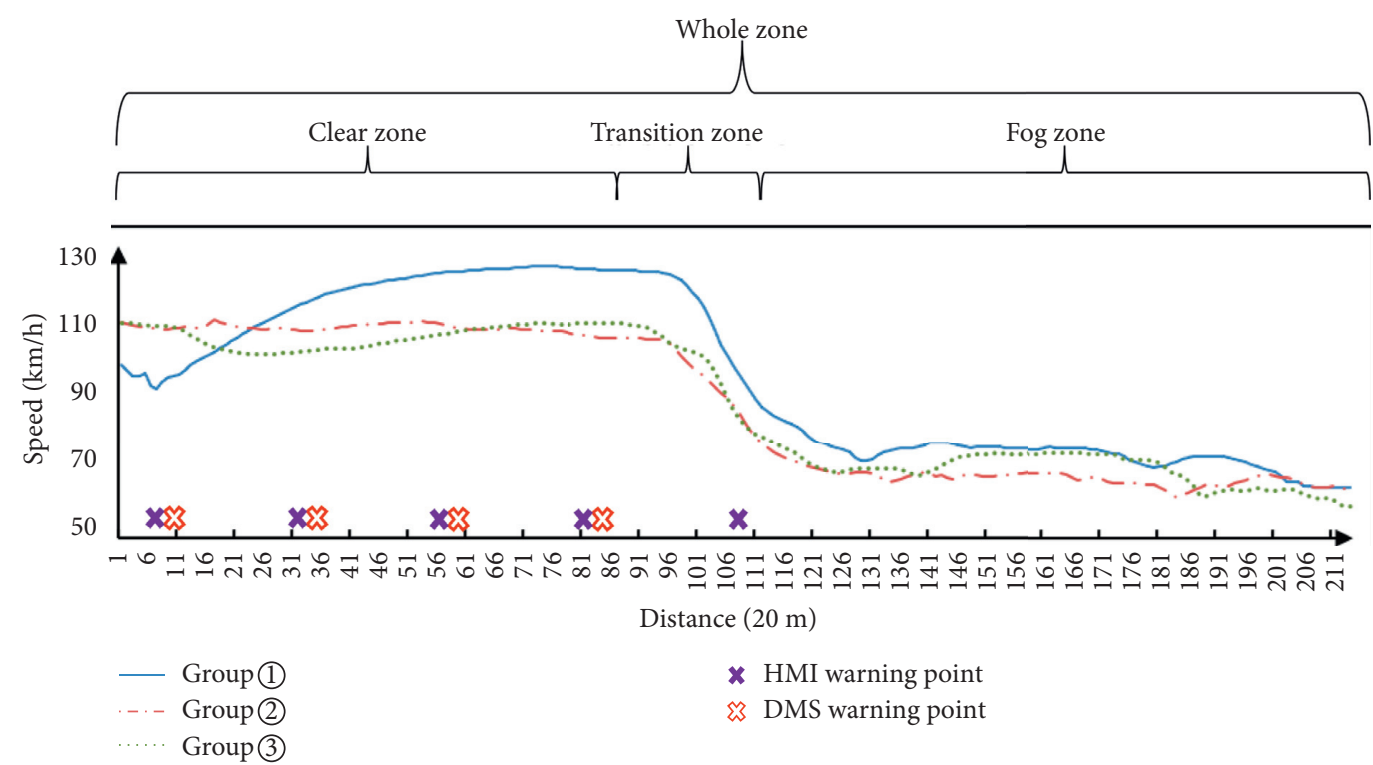

(a)
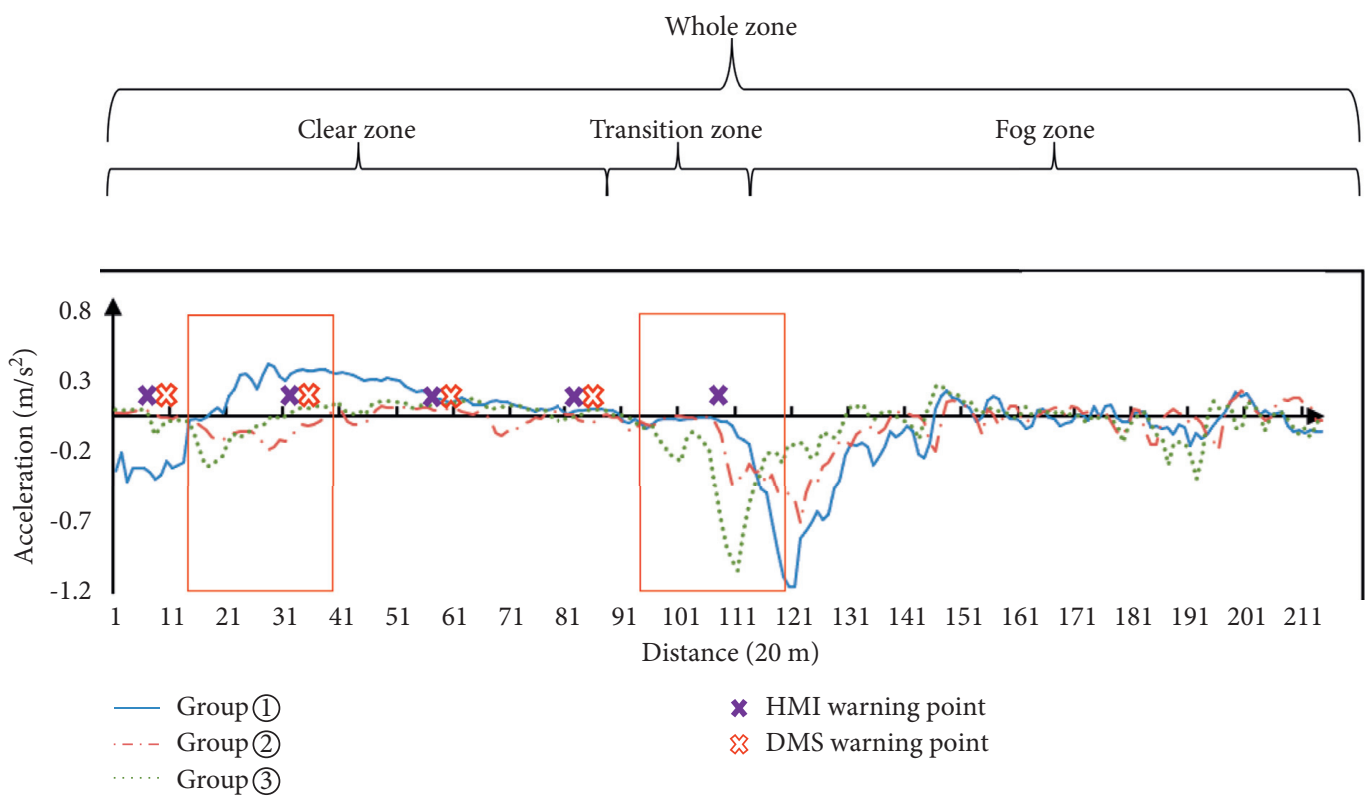

(b)

Figure 5: Heavy fog. (a) Speed and (b) acceleration. 


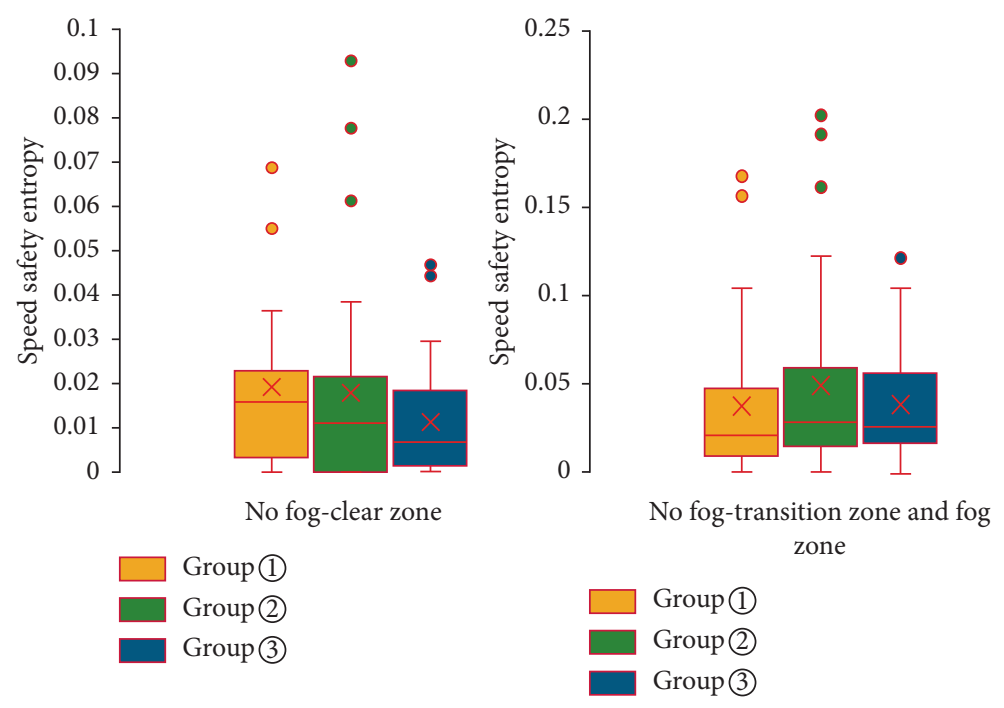

(a)
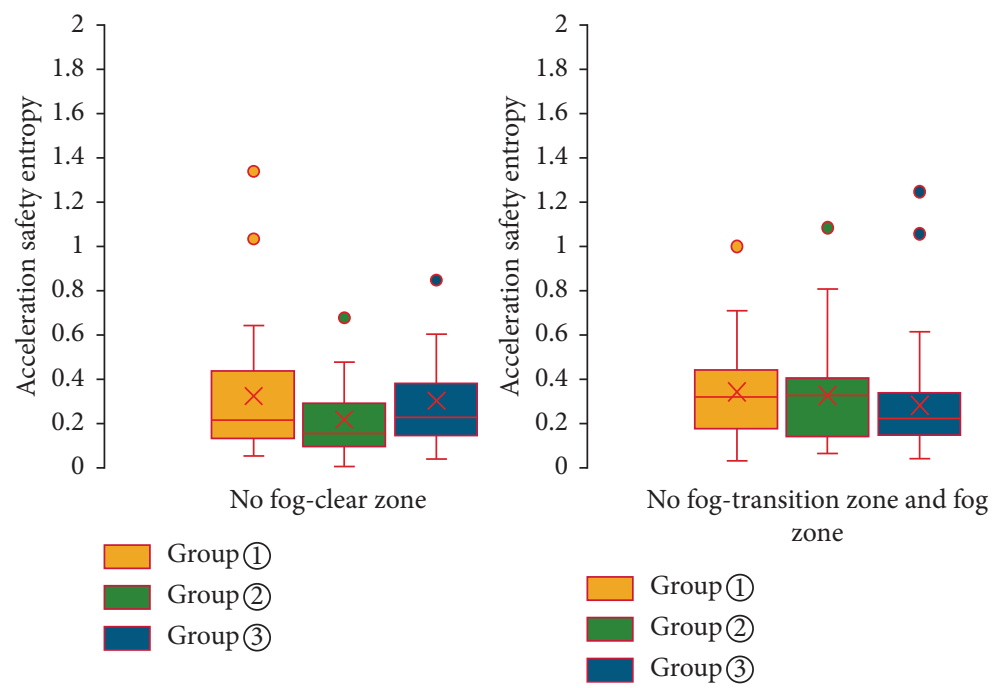

(b)

FIgURE 6: No fog. (a) Speed safety entropy in a no fog condition and (b) acceleration safety entropy in a no fog condition.

adjustment in group (3) is slightly lower, and the difference between individuals is smaller.

As for the acceleration safety entropy in heavy fog (Figure 8(b)), in the clear zone, the safety entropy value of acceleration increases significantly as the technical level increases, which indicates that the warning information of heavy fog has a great impact on driving behavior, and it has a negative impact on driving safety in the clear zone. In the transition zone and the fog zone, group (1) and group (2) exhibit small differences, but the safety entropy value in group (3) is significantly reduced, which indicates that the technical level of the fog warning system can effectively improve the driver's operational stability on foggy zones to improve the driving safety.

The above results indicate that the fog warning system has a positive effect on driving stability in heavy fog conditions. Before the visibility change, DMS is helpful to the orderly changes in the driver's speed. When the visibility starts to change, the fog warning with HMI seems to have a more positive effect on the driver's speed guidance.

\section{Discussion}

The goal of this study was to study the impact of a fog warning system on the driver's safety on foggy days. The fog warning system in this paper issued four times of warning messages in the warning zone, and the warning information included the concentration of fog and the distance from the fog zone. The results show that the fog warning system (DMS and HMI) can effectively assist the driver in speed control and improve driving safety on foggy days, but its impact on speed and acceleration is slightly different in different zones. Specifically, when the driver receives the warning information, they will take deceleration measures as the response. However, when the visibility has not changed, the driver's speed adjustment mode changes more frequently, and the 

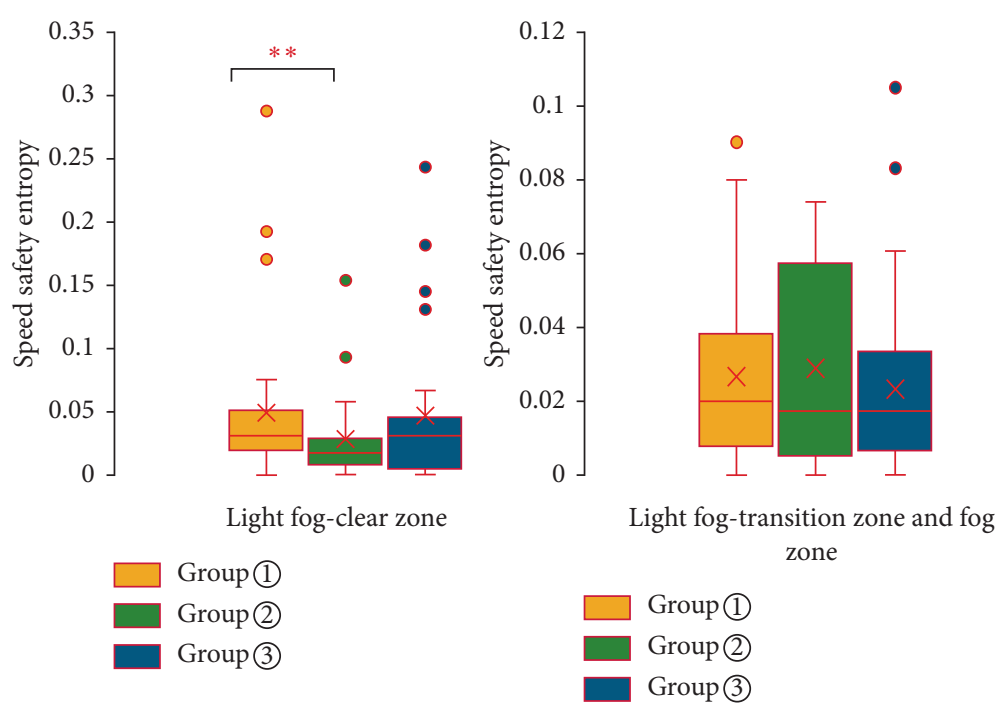

(a)
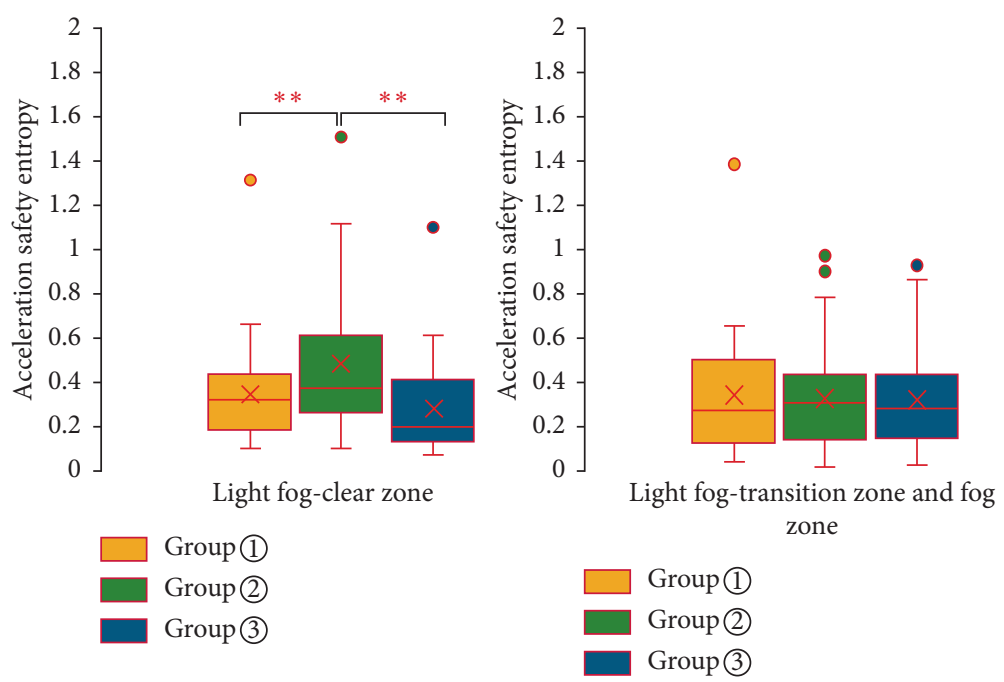

(b)

Figure 7: Light fog. (a) Speed safety entropy in a light fog condition and (b) acceleration safety entropy in a light fog condition. ** Significant at the $95 \%$ confidence level.

driver will accelerate to a certain extent due to the long distance to the fog. The ineffectiveness in reducing speed variability has also been mentioned in previous studies $[25,26]$. In the process of speed adjustment, HMI makes the driver decelerate earlier and faster, and the speed change process of drivers using DMS before visibility change is more orderly. In addition, warning messages are significant in reducing speeds in the fog zones. In the light fog zone, the average speed of the driving group using HMI is lower, and in the heavy fog zone, the average speed of the DMS group is lower. In general, a fog warning system is helpful to improve the safety of the driver in the fog zone.

Besides, this study conducted a predriving questionnaire survey and a postdriving questionnaire survey during the experiment. The predriving questionnaire survey is mainly designed to collect the basic information and the fatigue level of the driver. The purpose of the postdriving questionnaire survey is to understand the drivers' acceptance of DMS and HMI to supplement the objective data results. The results of the postdriving questionnaire showed that, under heavy fog conditions, the driver's acceptance rate for DMS was $83 \%$ and for HMI was 79\%; under light fog conditions, the driver's acceptance rate for DMS was $81 \%$ and for HMI was $76 \%$, which shows that the driver's acceptance of fog warning system is relatively high. The result is consistent with the previous research that drivers are more compliant with fog warning systems in heavy fog conditions $[27,28]$. In addition, it is undeniable that there are some disadvantages of driving simulation experiments. Participants may know that they are being recorded and observed, which could lead to some deviations in the collected driving behavior data. But this study used contrastive experiments to avoid this problem. Moreover, we performed subjective questionnaires on scene realism, speed perception, brake feel, and throttle 

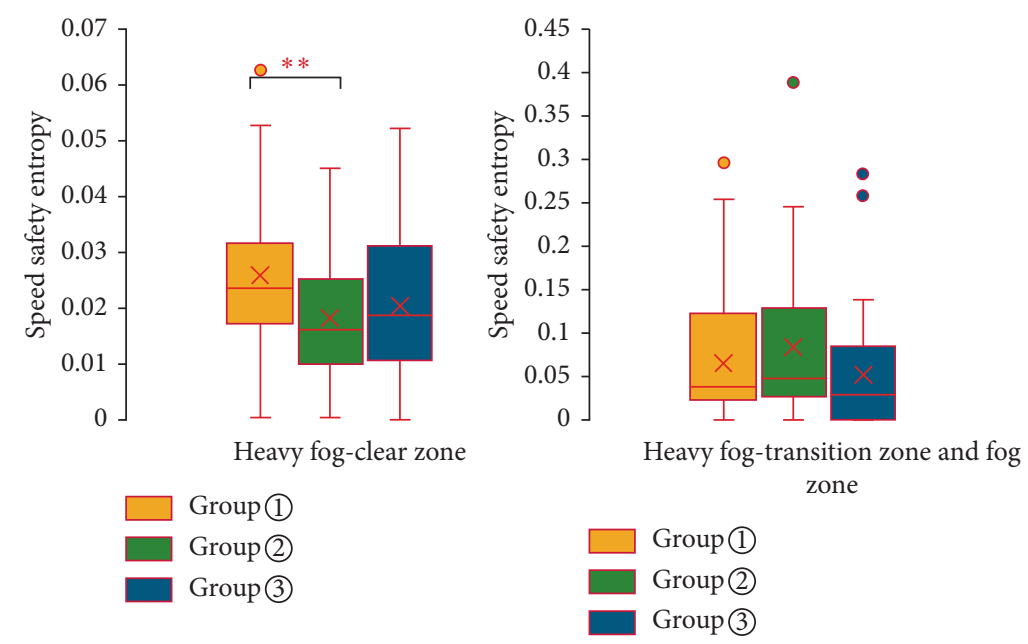

(a)
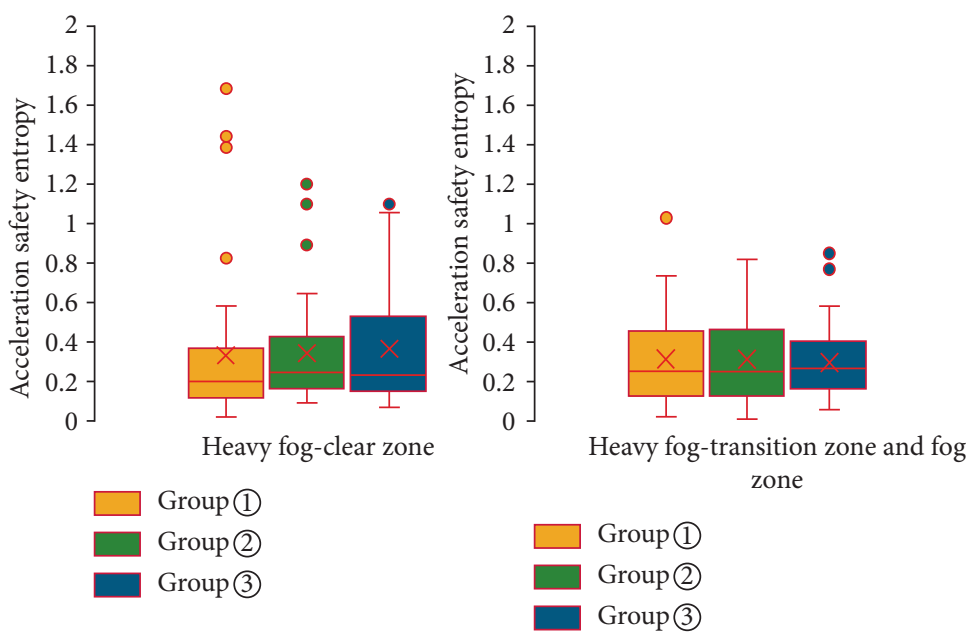

(b)

Figure 8: Heavy fog. (a) Speed safety entropy in a heavy fog condition and (b) acceleration safety entropy in a heavy fog condition. ** Significant at the $95 \%$ confidence level.

perception for all participants in the driving simulator experiment. The results showed that most drivers recognized the driving simulator, and the effectiveness of the driving simulator used in this study was verified in previous research [29].

In recent years, the Ministry of Transport in China has introduced a series of plans and policies to support CVrelated policy planning, standard formulation, technology research and development, and industrial landing. Testing the CV system based on human factors is the focus of research, including performance testing and service testing for users. Based on driving simulation technology, we conducted a test from the perspective of human factors to provide theoretical support for the practical application of the fog warning system. The findings can provide available evaluation indicators and general evaluation methods for the safety assessment of fog warning systems and provides a reference for the optimization design of these systems. It is helpful to understand the drivers' speed response behavior under different fog warning systems and provides a reference for the layout of the road-terminal DMS system and the information design of vehicle-terminal HMI.

Finally, some limitations should be noted. This study only selected two longitudinal indicators to analyze the driving safety influenced by fog warning systems. Other indicators could be combined to further evaluate the system, such as vehicle's lateral control and drivers' visual and psychological characteristics. Since it is a simulator study, a real vehicle test needs to be carried out in the future. In addition, it is necessary to further explore the influence of different design parameters of fog warning systems on driving behavior to provide better service for the system application.

\section{Conclusion}

The fog warning system can improve the speed stability and driving safety of drivers in fog zones. According to the results of the impact analysis, in conditions without risks (no fog), the HMI fog warning system can improve the driver's 
speed stability, while under fog conditions, the fog warning system makes the driver decelerate earlier, with a smaller average deceleration, before entering the fog zone. Thus, we can draw the conclusion that the higher the technical level is, the earlier the driver decelerates, so the system ensures the driver's safety in the fog zone. However, the existence of warning information may lead to a large fluctuation in speed after the warning, and this phenomenon is more obvious at a higher fog level.

According to the results of safety entropy, the fog warning system can improve the driver's speed stability in the entire fog zone; however, warning information may reduce the driving stability in the warning zone. In the light fog condition, the fog warning system with HMI has a better effect on improving speed stability and driving safety, while in the heavy fog condition, the two fog warning systems exhibit little difference.

This paper provides available evaluation indicators and general evaluation methods for the safety assessment of fog warning systems and provides a reference for the optimization design of these systems. In the future, real vehicle test is expected, and more indicators and the safety entropy of each indicator can be employed to comprehensively analyze the impact of fog warning systems. Except for the driving safety, the efficiency, ecology, comfort, and effectiveness of fog warning systems based on the driving simulation CV test platform need to be evaluated to achieve the comprehensive evaluation of the fog warning systems.

\section{Data Availability}

The data used to support the findings of this study are available from the corresponding author upon request.

\section{Conflicts of Interest}

The authors declare that there are no conflicts of interest regarding the publication of this paper.

\section{Acknowledgments}

This work was supported by the National Natural Science Foundation of China (52072012) and the State Key Laboratory of Automotive Safety and Energy under Project no. KF2017.

\section{References}

[1] B. Hamilton, J. G. Grabowski, B. Tefft, and L. Arnold, Hidden Highways: Fog and Traffic Crashes on America's Roads, AAA Foundation for Traffic Safety, Washington, DC, USA, 2014.

[2] National Bureau of Statistics of China, "Annual statistics on road traffic accidents," National Bureau of Statistics of China, Beijing, China, 2017, http://www.statsdatabank.com.

[3] K. L. M. Broughton, F. Switzer, and D. Scott, "Car following decisions under three visibility conditions and two speeds tested with a driving simulator," Accident Analysis and Prevention, vol. 39, 2007.

[4] S. Caro, V. Cavallo, C. Marendaz, E. R. Boer, and F. Vienne, "Can headway reduction in fog be explained by impaired perception of relative motion?," Human Factors: The Journal of the Human Factors and Ergonomics Society, vol. 51, no. 3, pp. 378-392, 2009.

[5] R. G. Hoogendoorn, G. Tamminga, S. P. Hoogendoorn, and W. Daamen, "Longitudinal driving behavior under adverse weather conditions: adaptation effects, model performance and freeway capacity in case of fog," in Proceedings of the 13th International IEEE Conference on Intelligent Transportation Systems, Funchal, Portugal, September 2010.

[6] A. S. Mueller and L. M. Trick, "Driving in fog: the effects of driving experience and visibility on speed compensation and hazard avoidance," Accident Analysis \& Prevention, vol. 48, no. 9, pp. 472-479, 2012.

[7] Q. Luo, X. Chen, J. Yuan, X. Zang, J. Yang, and J. Chen, "Study and simulation analysis of vehicle rear-end collision model considering driver types," Journal of Advanced Transportation, vol. 2020, Article ID 787, 11 pages, 2020.

[8] Q. Luo, X. Zang, J. Yuan, X. Chen, J. Yang, and S. Wu, "Research of vehicle rear-end collision model considering multiple factors," Mathematical Problems in Engineering, vol. 2020, Article ID 6725408, 11 pages, 2020.

[9] X. Zhao, W. Xu, J. Ma, H. Li, Y. Chen, and J. Rong, "Effects of connected vehicle-based variable speed limit under different foggy conditions based on simulated driving," Accident Analysis and Prevention, vol. 128, pp. 206-216, 2019.

[10] X. Chang, H. Li, L. Qin, J. Rong, Y. Lu, and X. Chen, "Evaluation of cooperative systems on driver behavior in heavy fog condition based on a driving simulator," Accident Analysis and Prevention, vol. 128, pp. 197-205, 2019.

[11] T. Zhao, Q. I. Chunhua, S. Zhu, M. Gao, and Y. Wang, "Study on influence of complexity of highway alignment on driver's HRV," China Safety Science Journal, vol. 11, 2016.

[12] W. A. N. Wei, W. Zhen-Hua, and W. Bao-Ju, "Research on detection of fatigue driving based on driving behaviors," Journal of Transportation Engineering, vol. 06, no. 16, pp. 21-24, 2016.

[13] M. Lei, C. Ke, W. Minhlu, and Q. Rui, "A study on driver behavior identification method under environment of vehicle-road integration," Automotive Engineering, vol. 40, no. 11, pp. 1330-1338, 2018.

[14] X. Zhao, Y. Chen, H. Li, J. Ma, and J. Li, "A study of the compliance level of connected vehicle warning information in a fog warning system based on a driving simulation," Transportation Research Part F: Traffic Psychology and Behaviour, vol. 76, pp. 215-237, 2021.

[15] X. Zhao, X. Li, Y. Chen, H. Li, and Y. Ding, "Evaluation of fog warning system on driving under heavy fog condition based on driving simulator," Journal of Intelligent and Connected Vehicles, vol. 4, 2021.

[16] D. Hu, X. Feng, X. Zhao, H. Li, J. Ma, and Q. Fu, "Impact of HMI on Driver's Distraction on a Freeway Under Heavy Foggy Condition Based on Visual Characteristics," Journal of Transportation Safety \& Security, pp. 1-24, 2020.

[17] Certification and Accreditation Administration of the People's Republic of China, Regulations for the Implementation of the Road Traffic Safety Law of the People's Republic of China, Certification and Accreditation Administration of the People's Republic of China, Beijing, China, 2017.

[18] J. I. Ge and G. Orosz, "Dynamics of connected vehicle systems with delayed acceleration feedback," Transportation Research Part C: Emerging Technologies, vol. 46, no. 9, pp. 46-64, 2014.

[19] J. Lerman, "Study design in clinical research: sample size estimation and power analysis," Canadian Journal of Anaesthesia, vol. 43, no. 2, pp. 184-191, 1996. 
[20] S.-C. Chow, J. Shao, H. Wang, and Y. Lokhnygina, Sample Size Calculations in Clinical Research: Third Edition, Chapman and Hall/CRC Biostatistics Series, New York, NY, USA, 2017.

[21] SAC, Grade of Fog Forecast, Standardization Administration of the People's Republic of China, Beijing, China, 2012.

[22] J. S. Richman and J. R. Moorman, "Physiological time-series analysis using approximate entropy and sample entropy," American Journal of Physiology-Heart and Circulatory Physiology, vol. 278, no. 6, pp. H2039-H2049, 2000.

[23] D. E. Lake, J. S. Richman, M. P. Griffin, and J. R. Moorman, "Sample entropy analysis of neonatal heart rate variability," American Journal of Physiology-Regulatory, Integrative and Comparative Physiology, vol. 283, no. 3, pp. R789-R797, 2002.

[24] X. Zhao, X. U. Shili, J. Rong, and X. Zhang, "Discriminating Threshold of Driving Fatigue Based on the Electroencephalography Sample Entropy by Receiver Operating Characteristic Curve Analysis," Journal of Southwest Jiaotong University, vol. 48, 2013.

[25] A. S. Al-Ghamdi, "Experimental evaluation of fog warning system," Accident Analysis and Prevention, vol. 39, no. 6, 2007.

[26] L. N. Boyle and F. Mannering, "Impact of traveler advisory systems on driving speed: some new evidence," Transportation Research Part C: Emerging Technologies, vol. 12, 2004.

[27] H. M. Hassan and M. A. Abdel-Aty, "Analysis of drivers' behavior under reduced visibility conditions using a Structural Equation Modeling approach," Transportation Research Part F: Traffic Psychology and Behaviour, vol. 14, no. 6, pp. 614-625, 2011.

[28] C. A. MacCarley, C. Ackles, and T. Watts, "A study of the response of highway traffic to dynamic fog warning and speed advisory messages," in Proceedings of the 85th Annual Meeting Transport Research Board, Washington, DC, USA, 2006.

[29] H. Ding, X. Zhao, J. Rong, and J. Ma, "Experimental research on the effectiveness of speed reduction markings based on driving simulation: a case study," Accident Analysis and Prevention, vol. 60, no. 1, pp. 211-218, 2013. 